\title{
Deaf intermarriage does not increase the prevalence of deafness alleles
}

2 Derek C. Braun $^{1^{*}}$, Samir Jain ${ }^{1 \mathscr{I}}$, Eric Epstein ${ }^{1 \mathscr{I}}$, Brian H. Greenwald ${ }^{2 \&}$, Brienna Herold ${ }^{1 \&}$, and

3 Margaret Gray ${ }^{1 \&}$

4

$5{ }^{1}$ Department of Science, Technology, and Mathematics, Gallaudet University, Washington, D.C.

$6{ }^{2}$ Department of History, Philosophy, Religion, and Sociology, Gallaudet University,

7 Washington, D.C.

8

9 IThese authors contributed equally to this work.

$10{ }^{\&}$ These authors also contributed equally to this work.

11

$12 *$ Corresponding author

13 E-mail: derek.braun@gallaudet.edu (DB) 


\section{Abstract}

The idea that deaf intermarriage increases deafness was forcefully pushed in the late 19th century

17 by Alexander Graham Bell, in proceedings published by the National Academy of Science.

18 Bell's hypothesis was not supported by a 19th century study by Edward Allen Fay, which was

19 funded by Bell's own organization, the Volta Bureau. The Fay study showed through an analysis

20 of 4,471 deaf marriages that the chances of having deaf children did not increase significantly

21 when both parents were deaf. In light of an apparent increase in non-complementary pairings

22 when a recent dataset of Gallaudet alumni was compared with the 19th century Fay dataset,

23 Bell's argument has been resurrected that residential schools for the deaf, which concentrate

24 signing deaf individuals together, have promoted assortative mating and increased the prevalence

25 of both phenotypic deafness and the commonest recessive deafness allele. Because this

26 hypothesis persists, even though it contradicts classical models introduced by R.A. Fisher and

27 Sewell Wright, it is critically important that this hypothesis be thoroughly re-investigated. In this

28 study, we used an established forward-time genetics simulator with parameters and

29 measurements collected from the published literature. Compared to mathematical equations,

30 simulations allowed for more complex modeling, operated without assumptions of parametricity,

31 and captured ending distributions and variances. Our simulation results affirm predictions from

32 classical equations and show that assortative mating only modestly increases the prevalence of

33 phenotypically deaf individuals, with this effect mostly completed by the third generation. Most

34 importantly, our data show that even intense assortative mating does not increase allelic

35 frequency under reported conditions. These results are not locus-specific and are generalizable to

36 other forms of recessive deafness. We offer alternative explanations for the higher rate of non-

37 complementary pairings measured in the contemporary Gallaudet alumni sample as compared to

38 the Fay dataset.

39 Introduction

40 In an 1883 presentation to the National Academy of Sciences, Alexander Graham Bell delivered

41 an ominous warning about the intermarriage of deaf individuals [1]. If intermarriage was left 
42 unchecked, Bell argued, this would lead to a "deaf variety of the human race." Bell delineated

43 the costs of educating deaf individuals and argued that the residential schools were an economic

44 burden to state governments funding the schools [1]. Following this address, Bell conducted

45 research on hereditary deafness on Martha's Vineyard in the late 1880s. Bell persisted in efforts

46 to better understand the transmission of genetic deafness, although he ultimately never

47 understood it [2,3]. To this end, he hired Edward Allen Fay, who was the vice-president at

48 Gallaudet College and editor of the American Annals of the Deaf. Bell's Volta Bureau funded

49 Fay's landmark study of 4,471 deaf marriage pedigrees, collected from alumni of Gallaudet

50 University, whose students are predominantly deaf, and alumni from residential schools for the

51 deaf throughout the United States. Although the Fay study eventually concluded that deaf

52 intermarriage did not much increase the chances of having deaf children [4], Bell remained vocal

53 in his beliefs that the ideal marriage was a marriage between a deaf and hearing person. As the

54 wealthy inventor of the telephone, he remained highly influential in the scientific community and

55 in the nascent eugenics movement.

56 The results of Fay's study were not well understood at the time because they were published in

57 1898, before the rediscovery of Mendel's work [5] and the many discoveries in experimental and

58 theoretical genetics that followed. In those days, the understanding of the heredity of deafness

59 and heredity in general was based on observation, such as those recorded by the otologist

60 William Wilde in 1857, or by tallying summary statistics, like Fay’s work [6].

61 We now know that genetic deafness [OMIM 220290] accounts for the majority of deafness in

62 children and is caused by mutations in >140 already mapped genes [7]. Of these, genetic

63 deafness due to connexin 26 (GJB2) variant alleles [OMIM 121011] is by far the most common,

64 accounting for more than a quarter of congenital deafness [8]. Three GJB2 frameshift variants

65 account for most severe to profound congenital deafness, and are associated with specific ethnic

66 groups: c.35delG in European ancestry, c.167delT in Ashkenazi Jewish ancestry, and c.235delC

67 in Asian ancestry [9-11]. These three variants are inherited in an autosomal recessive fashion,

68 and cause nonsyndromic deafness, meaning that there are no other discernible physical

69 characteristics.

70 In fact, most deaf intermarriage produces hearing children mainly because of the

71 complementation between the many recessive genes causeing deafness, and also because 
72 deafness often occurred or was observed after birth and was usually attributed to injury or

73 childhood morbidity. Fay [4] termed these cases as "adventitious."

74 In the early 20th century, R.A. Fisher [12] and Sewell Wright [13] introduced a mathematical

75 model for inbreeding that could be applied to assortative mating, reworked by Crow and

76 Felsenstein [14]. In a large population and in the absence of selective pressure, assortative

77 mating increases the phenotypic expression of recessive alleles, but the allelic frequencies

78 themselves do not change. Therefore, deaf intermarriage could be expected to cause an initial

79 and limited increase in phenotypic deafness, and would have no effect on the allelic frequency

80 [14].

81 In contradiction to Fisher's and Wright's classical model, recent authors have posited that

82 assortative mating between signing deaf individuals who socialized together in residential

83 schools over slightly more than 200 years in the United States has increased both phenotypic and

84 allelic frequencies for recessive deafness [15,16]. Termed "linguistic homogamy," it is reasoned

85 to be motivated by an innate human need for easy and effective communication. Signing deaf

86 individuals would find linguistically compatibility in one another and intermarry. This

87 hypothesis was used to explain results from a pedigree study by Arnos et al. [17] which had

88 shown that non-complementary pairings in a contemporary Gallaudet alumni dataset were

89 occurring more often than non-complementary pairings in the original Fay dataset, which had

90 been collected more than 100 years earlier.

91 The degree of assortative mating among deaf Americans has been measured, and has been

92 relatively stable over the past 200 years. Fay [4], in 1898, initially reported an uncorrected

93 measure of $72.5 \%$. From the 1970 National Census of the Deaf Population (NCDP) in the United

94 States, Schein and Delk [18] calculated a figure of 80-90\%. Most recently. Blanton et al. [19]

95 calculated a figure of $79 \%$ from a sample of Gallaudet alumni, who are predominantly white

96 Americans.

97 The reproductive fitness of deaf individuals has also been investigated through fertility. All

98 reports from the literature report markedly depressed relative fitness. Values normalized against

99 the general population range from 0.31 to 0.91 . The highest measured fitness of 0.91 , which is

100 still depressed, was reported from an educated American deaf sample from Gallaudet University

101 [18-22]. 
102 This continuing question of whether assortative mating between deaf individuals affects the

103 incidence of phenotypic deafness and the prevalence of deafness alleles is important because it

104 has potential implications for policy and funding decisions. This includes popular support for

105 funding of residential deaf education programs, which bring deaf people together into social

106 groups. It may also alter opinions about eugenics, which was popular in fairly recent history,

107 particularly in Germany and in Scandinavian countries. Discussion about the ethics of eugenics

108 is moving to the forefront again, given the recent use of gene-editing technologies (CRISPR) in

109 humans $[23,24]$. It is therefore critically important that the question "does deaf intermarriage

110 (assortative mating) increase the prevalence of deafness" is carefully examined using a variety of

111 approaches.

112 In this study, we performed thousands of forward-time evolutionary simulations using an

113 established package [25,26]. We simulated assortative mating and measured the changes in

114 phenotypic deafness and allelic frequencies, using parameters scoured from the published

115 literature. We tested the hypothesis that assortative mating influences the gene pool and used

116 statistical analyses to compare median results and ending distributions. This approach allowed us

117 to capture the variance in end results without any underlying assumptions of parametricity. We

118 further compared these results with mathematical modeling.

\section{Results}

120 We initially ran 5,000 simulations following parameters established by Nance and Kearsey [15]

121 so that we could directly compare our simulation results with theirs. First, the initial allelic

122 frequency of the recessive deafness allele was set to $1.304 \%$. This frequency is approximately at

123 the midpoint of the $0.6 \%$ to $3.5 \%$ reported range of carrier frequencies for the c.35delG variant

124 in $G J B 2$ in white Americans and Europeans $[9,27,28]$. Simulations were run over 20 generations

125 (400 years), which reflects the approximate time frame that signed languages are believed to

126 have existed, deaf individuals have formed close social ties, and assortative mating among deaf

127 individuals has been occuring [15]. The constant population size was set to 200,000 simulated

128 individuals [15]. At each generation, a proportion of simulated hearing individuals were

129 randomly selected (of which a small proportion, by random choice, carried a single recessive

130 deafness allele), and assigned phenotypic deafness at a conservative rate of 0.8 per 1,000

131 simulated individuals, which is a measured frequency of profound deafness at birth [29,30]. This 
132 assigned deafness, biologically, reflects genetic deafness due to other, complementary genes,

133 deafness from epigenetic causes, and/or perinatal morbidity. Although the prevalence of

134 identified deafness continues to increase throughout childhood to approximately 3.5 per 1,000

135 [30], this higher figure was not used in our simulations. Genetically deaf simulated individuals

136 and simulated individuals with assigned deafness from other causes were mated together in the

137 same pool. For our initial analysis, assortative mating was set to $0 \%$ and $90 \%$ to create two

138 datasets for endpoint comparison; in subsequent analyses, simulations were run over a range of

139 degrees of assortative mating. The 90\% assortative mating figure is per Nance and Kearsey [15];

140 see Background for further detail.

141 Under these parameters, after 20 generations (400 years), the median frequency of deaf

142 individuals with our recessive allele increased by $23 \%$ relative to the simulations with no

143 assortative mating, that is, $0.017 \%$ as compared to $0.0220 \%$, which is statistically significant

144 (Fig 1 and Table 1; $n=5,000$ simulations, Mann-Whitney $U=5.83 \times 10^{6}, p<10^{-308}$, common

145 language effect size $f=76.68 \%$ ). This statistic was identical to the calculation of $0.0220 \%$ using

146 equation (3) from Crow and Felsenstein [14] (Table 1) and described in Materials and Methods.

147 Most of the change occurred within the first three generations. This figure is 7-fold less than the

$148 \sim 0.16 \%$ frequency reported elsewhere in a comparable simulation of deaf-deaf assortative mating

149 with essentially the same parameters [15].

150 The frequency of the recessive deafness allele did not increase significantly; it was $1.304 \%$

151 versus $1.306 \%$ after 20 generations (Fig 1 and Table $1 ; n=5,000$ simulations, Mann-Whitney

$152 U=1.25 \times 10^{7}, p=0.94$, common language effect size $f=49.96 \%$ ). Likewise, this figure is also

153 much less than the $\sim 1.7 \%$ frequency reported elsewhere in a simulation of deaf-deaf assortative

154 mating with essentially the same parameters [15].

155 The inbreeding coefficient, $F$, was different: 0 versus 0.00376 , which was statistically significant 156 ( $n=5,000$ simulations, Mann-Whitney $U=4.98 \times 10^{6}, p<10^{-308}$, common language effect size

$157 f=80.09 \%$. This increase in $F$ was small since it was being attenuated because of competition

158 for mates between the small number of simulated genetically deaf individuals and the larger pool

159 of simulated individuals with assigned deafness due to other causes. 
161 Fig 1. Effect over time of assortative mating on the frequencies of genetically deaf

162 individuals and a recessive deafness allele. Lines, from top to bottom, represent a five-number

163 summary: $98 \%$ percentile, $75 \%$ quartile, median, $25 \%$ quartile, and $2 \%$ percentile. To the right

164 of each subplot is a violin plot showing the distribution of the endpoint data. The tips of the

165 violins represent the extrema. The vertical lines within the violins show the $2 \%$ through $98 \%$

166 percentile. The boxes within the violins show the first through third quartile. The cross-hatches

167 show the medians. Simulations were run with relative fitness $=1.0$ and other parameters as

168 described in Materials and Methods.

170 We next ran simulations over a range of degrees of assortative mating (Fig 2 and Table 1). The

171 frequency of deafness increased proportionately to assortative mating. Most of the change

172 occurred in the first three generations. All differences in the frequency of deafness between each

173 endpoint were highly significant with all $p<10^{-40}$. The results also in close agreement with

174 calculations using equation (3) from Crow and Felsenstein [14] (Table 1) and described in

175 Materials and Methods. The allelic frequency, however, remained invariable regardless of the

176 extent of assortative mating, and no statistically significant difference was found between any of

177 the endpoints $\left(n=5,000\right.$ simulations, Kruskal-Wallis $H=1.5, p=0.69$, effect size $\eta^{2} \approx 0.0 \%$ ).

Fig 2. Effect of assortative mating on the frequencies of genetically deaf individuals and a recessive deafness allele. Violin plots show the distributions of the endpoint data after 20

181 generations. The tips of the violins represent the extrema. The vertical lines within the violins

182 show the $2 \%$ through $98 \%$ percentile. The boxes within the violins show the first through third 183 quartile. The cross-hatches show the medians. Simulations were run with relative fitness $=1.0$ 184 and other parameters as described in Materials and Methods. 


\section{Synergistic Effects of Fitness And Assortative Mating on Allelic} 187 Frequency

188 Because assortative mating increases the phenotypic expression of alleles, it would therefore

189 modulate the effects of selective pressure upon those alleles. Nance and Kearsey [15] have

190 argued that relaxed fitness would be necessary for increasing the numbers of deaf individuals.

191 We therefore simulated assortative mating across a range of relative fitnesses.

192 The frequency of the recessive deafness allele was sensitive to relative fitness, which became

193 particularly noticeable at or above fitnesses of 1.5 when combined with assortative mating. (Fig

1943 and Table 1).

196 Fig 3. Synergy of assortative mating and fitness on the frequencies of genetically deaf

197 individuals and a recessive deafness allele. Right: no homogamy; left: 90\% homogamy. Violin

198 plots show the distributions of the endpoint data after 20 generations. The tips of the violins

199 represent the extrema. The vertical lines within the violins show the $2 \%$ through $98 \%$ percentile.

200 The boxes within the violins show the first through third quartile. The cross-hatches show the 201 medians.

202

203

Deaf Individuals

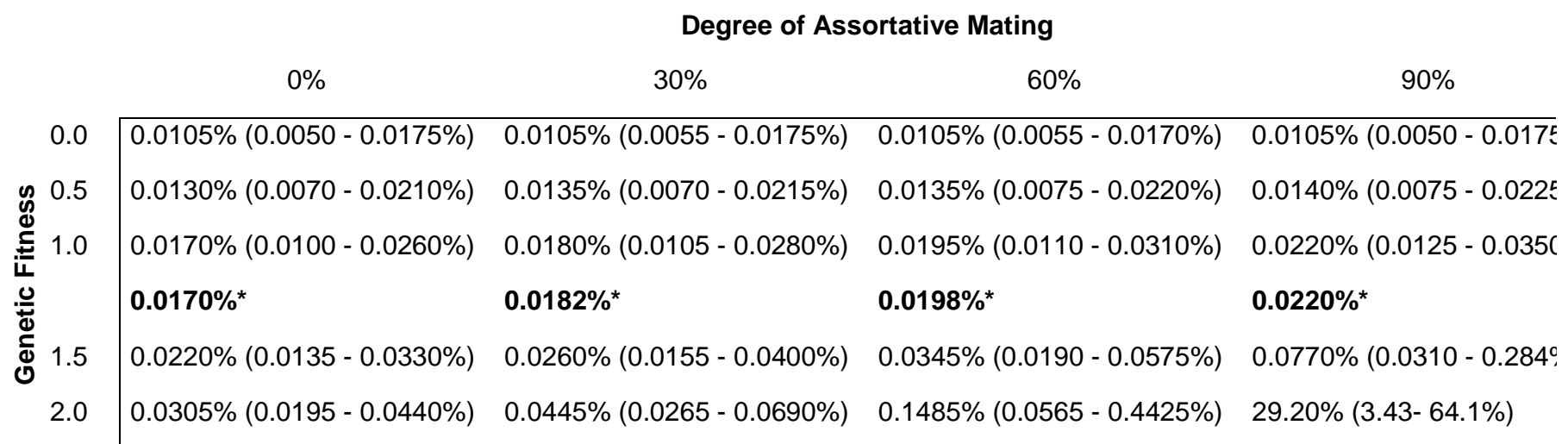


Degree of Assortative Mating

\begin{tabular}{|c|c|c|c|c|}
\hline & $0 \%$ & $30 \%$ & $60 \%$ & $90 \%$ \\
\hline 0.0 & $1.03 \%(0.908-1.16 \%)$ & $1.03 \%(0.912-1.16 \%)$ & $1.03 \%(0.910-1.16 \%)$ & $1.03 \%(0.909-1.16 \%)$ \\
\hline 0.5 & $1.15 \%(1.02-1.30 \%)$ & $1.15 \%(1.01-1.30 \%)$ & $1.15 \%(1.01-1.29 \%)$ & $1.14 \%(1.00-1.28 \%)$ \\
\hline$=1.0$ & $1.30 \%(1.15-1.47 \%)$ & $1.30 \%(1.15-1.47 \%)$ & $1.30 \%(1.15-1.47 \%)$ & $1.31 \%(1.15-1.47 \%)$ \\
\hline 1.5 & $1.49 \%(1.31-1.70 \%)$ & $1.52 \%(1.33-1.74 \%)$ & $1.56 \%(1.35-1.81 \%)$ & $1.70 \%(1.41-2.22 \%)$ \\
\hline 2.0 & $1.74 \%(1.52-2.00 \%)$ & $1.86 \%(1.60-2.17 \%)$ & $2.32 \%(1.78-3.41 \%)$ & $36.4 \%(6.17-73.3 \%)$ \\
\hline
\end{tabular}

Table 1. Simulation results showing effects of assortative mating and fitness on the

frequency of deafness after 200 years. Values given are medians, with $2 \%$ through $98 \%$

percentiles in parentheses. Values in bold and followed by an asterisk were calculated from

210 equation (3) from Crow and Felsenstein [14] as described in Materials and Methods. Simulations

211 were run as described in Materials and Methods.

\section{Discussion}

213 In this study, we addressed the century-old debate about whether deaf intermarriage increases the

214 phenotypic expression of genetic deafness. We also investigated a recent claim, based on

215 simulations, that assortative mating could increase the allelic frequency of the commonest

216 deafness allele, which is recessive $[15,16]$. We ran forward-time computer simulations using the

217 simuPOP package, which has been used by others for assortative mating simulations, to test the

218 hypothesis that assortative mating among deaf Americans (deaf intermarriage; linguistic

219 homogamy) would affect phenotypic deafness as well as allelic frequencies for recessive

220 deafness [25,26]. For each scenario, we analyzed the results of 5,000 simulations. We used

221 statistical analyses to compare not only median results, but also ending distributions. We used

222 this approach in addition to mathematical modeling because it allowed us to capture the variance

223 in results without underlying assumptions of parametricity, and allowed us to easily set up the

224 more complex scenario of introducing individuals with acquired or complementary deafness to

225 the mating pool.

226 Our simulations confirm that intense (90\%) assortative mating would increase the frequency of

227 homozygous deaf individuals by $23 \%$ over 20 generations. Most of this increase would occur

228 within the first three generations of assortative mating. However, the simulations also confirmed 
229 that assortative mating did not affect allelic frequencies at all (Fig 1 and 2 and Table 1). These

230 simulation results are consistent with predictions from classical genetics models and in nearly

231 identical agreement with mathematical calculations using Crow and Felsenstein's [14] equation

232 (3) (Table 1). Our results did not match with the results of a computer simulation published

233 elsewhere using essentially the same parameters, which predicted a $\sim 7$-fold increase in the

234 number of homozygous deaf individuals, as well as a $~ 30 \%$ increase in the frequency of the

235 recessive deafness allele [15].

236 Our simulation results also confirm that relative reproductive fitness impacts allelic frequencies.

237 Because assortative mating increases the expression of rare alleles, relative fitness acted

238 synergistically with assortative mating in our simulations to accelerate changes in recessive

239 allelic frequencies. In our simulations with greatly exaggerated relative fitness (1.5x and 2x),

240 allelic frequencies increased. However, these results do not apply to the worldwide deaf

241 community today because there are no reports in the literature of any population with higher-

242 than-normal relative fitness for deaf individuals. Instead, the literature uniformly reports

243 depressed relative fitness, ranging from 0.31 to 0.91 ; the highest measured fitness of 0.91 , which

244 is still depressed, was from an educated American deaf sample from Gallaudet University [18-

$24522]$. Therefore, based on our simulations, the synergy of assortative mating with depressed

246 fitness should be expected to constrain the frequency of recessive deafness alleles to low levels

247 (Fig 3 and Table 1).

248 Nance [15] forwarded a hypothesis that assortative mating based on shared language

249 compatibility, which he termed linguistic homogamy, among early humans may have accelerated

250 the evolution of human speech genes, particularly FOXP2, some 150,000 years ago. Nance

251 argued that improved language skills in early humans likely correlated with better cooperation,

252 better survival and higher fitness. Our simulation results support this intriguing hypothesis.

253 Preferential mating among those with advantageous language capabilities would have increased

254 the expression of these alleles and synergistically accelerated this evolution.

255 In 2008, Arnos and colleagues [17] studied contemporary pedigrees collected from Gallaudet

256 University alumni, and compared them with pedigrees from Fay's study, which were ascertained

257 from deaf institutions across the country. Segregation analysis comparing these two datasets

258 showed that the proportion of non-complementary pairings were $4.2 \%$ in the 1801-1899 Fay 
dataset and $23 \%$ in the 2007 Gallaudet alumni dataset. Our simulation results show that assortative mating among the larger deaf community is not a sufficient explanation to explain all of this difference in non-complementary pairings between these two datasets. Rather, we agree with the authors' deliberations that the reason may lie in the difference between how these two datasets were ascertained [17]. The original Fay dataset were collected from deaf institutions across the country, whereas the newer dataset were collected only from Gallaudet alumni. The Gallaudet dataset likely represents a special subpopulation. Gallaudet, established in 1864, is the world's only university for deaf students. Approximately $95 \%$ of its undergraduate student body are deaf. Today, Gallaudet attracts legacy students from multigenerational deaf families, whose parents and, in some cases, grandparents had previously attended Gallaudet. While deaf individuals at the time of the original Fay dataset in 1890 were usually from hearing families and were themselves discouraged from marrying others from deaf families, there is a noted preference for contemporary individuals from deaf families to attend Gallaudet and to pair with others from deaf families because of cultural and linguistic compatibility. Genetically deaf

273 individuals with multigenerational pedigrees, concentrated into the Gallaudet community and

274 directly seeking out one another, bypasses competition from those with acquired deafness,

275 increases the odds of non-complementation, and accelerates the phenotypic effects of assortative

276 mating. However, (1) this is only happening within communities like Gallaudet's which are

277 enriched for multigenerational deaf families, and does not reflect the genetic assortment of deaf

278 individuals in the greater population; (2) the increase in phenotypic expression had already

279 mostly occurred after the first three generations of intensive assortative mating and would be 280 predicted to have tapered off already; and (3) regardless of the intensity of assortative mating, it

281 has had no effect on the prevalence of deafness alleles in the overall population.

282 We are left with the puzzling paradox of how the commonest GJB2 variant alleles causing severe 283 to profound deafness: c.35delG, c.167delT, and c.235delC, have been measured at prevalences of

284 between $1 \%$ and $4.4 \%$, while measurements of reproductive fitness in deaf communities have

285 been uniformly depressed [9-11,18-22]. These three frameshift alleles account for the majority of 286 severe to profound nonsyndromic deafness in white Americans [9-11]. One possibility is

287 mutation-selection equilibrium: novel GJB2 mutations are perhaps being introduced at the same

288 rate that mutations in the gene pool are being eliminated. Evidence showing a mutational hotspot 289 at GJB2, particularly for deletion mutations, would provide support for this hypothesis. A 
290 second, and intriguing possibility is that of balancing selection. Unrelated to studying deafness,

291 Tran van Nhieu, Clair et al. [31] have shown in tissue culture experiments that Shigella flexneri

292 requires GJB2 connexons for egression into the intestinal epithelia, raising the possibility that the

293 three common GJB2 deletions could confer resistance to dysentery.

294 Connexons are dimers of hexameric proteins made up of individual connexins; in individuals

295 with GJB2 deletions, GJB2 is replaced by other connexons to form connexins which appear to

296 retain normal function everywhere except for the cochlea. Dysentery has been endemic at least

297 since the advent of urbanization, and resistance to this disease via altered connexons may have

298 provided enough positive selection to bring the commonest GJB2 mutations to their present

299 frequencies. This hypothesis is intriguing and should be investigated. Further, it would be

300 interesting to see if this advantage exists only for GJB2 variant homozygotes, or if heterozygous

301 carriers for recessive GJB2 deafness would also be resistant to shigellosis.

302 We hope that this study can put to rest the century-long argument put forth by Alexander

303 Graham Bell [1] that deaf intermarriage increases deafness in the gene pool. Using simulations,

304 and drawing upon mathematical modeling, with measurements and parameters collated from the

305 published literature over more than a century of data, our results unequivocally affirm the

306 classical models introduced by R.A. Fisher [12] and Sewell Wright [13]. That is, our data show

307 that while deaf intermarriage initially had some effect on the incidence of phenotypic deafness,

308 this effect was mostly completed by the third generation of assortative mating. In the time frame

309 of American deaf institutions, this effect would have completed approximately around the end of

310 the $19^{\text {th }}$ century, when Fay [4] collected and reported his data. However, deaf intermarriage and

311 assortative mating did not, and will not, change the prevalence of recessive deafness alleles

312 unless there is strong positive selection present. Therefore, Alexander Graham Bell's [1] "deaf

313 variety of the human race" will not happen even if deaf intermarriage and assortative mating

314 continue at this rate. 


\section{Materials and Methods}

\section{Code and Dataset}

317 The source code and dataset created for this study are publicly available from

318 https://github.com/derekbraun/homogamy.git so that anyone can replicate our experiments and

319 build upon our work.

\section{Simulations}

321 Simulations were performed using simuPOP 1.1 .10 .8 which is a forward-time population

322 genetics package, scriptable via Python, that has been used to simulate assortative mating

$323[25,26]$. Simulations were scripted with Python 3.7 .4 on a computer running macos

32410.14 .6. Simulations were parallelized on a 16-core Intel Xeon workstation. It required 80

325 hours of CPU time to complete the final simulations shown in this manuscript. We modeled both

326 assortative mating (homogamy) and reproductive fitness using a non-monogamous mating

327 scheme. Non-monogamous mating was chosen, after some experimentation with code, because

328 this allowed for better stability in the final proportion of homogamy per generation given the

329 small number of deaf individuals in the simulated population. Sexes were not assigned to

330 individuals; this was decided, after some experimentation with code, because it simplified coding

331 and sped up execution time.

332 After each generation, the following was calculated: the frequencies of the dominant and

333 recessive alleles $\mathrm{A}$ and $\mathrm{a}$; the frequencies of the homozygous dominant, heterozygous, and

334 homozygous recessive genotypes AA, Aa, and aa; the number of individuals with each genotype;

335 the number and frequency of deaf individuals (including acquired deafness); and the inbreeding

336 coefficient $(F)$ calculated as follows:

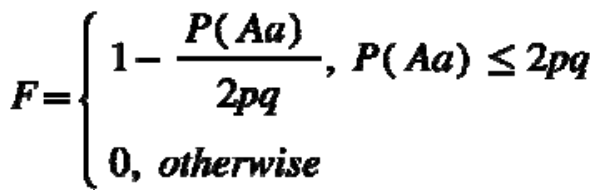

338 The frequency of deafness alleles from simulations were also compared to those calculated from

339 equation (3) of Crow \& Felsenstein [14]. The effective assortative mating fraction, $r$, was

340 derived from the \% assortative mating (homogamy) and re-estimated after each generation by

341 adjusting it by the size of the mating pool. This calculation matches the logic used in the forward 
342 simulation script which is that the initial mating pool size included all forms of profound

343 deafness at the rate of 0.8 per 1,000 individuals [29]. Therefore, initially and before assortative

344 mating, $q^{2}$ individuals have genetic deafness due to connexin 26 , and $0.008-q^{2}$ individuals have

345 acquired or complementary genetic deafness. At $t_{0}, R_{t}=q^{2}$, so at $t_{0}$, the expression for the mating

346 pool size, $0.008-q^{2}+R_{t}$ simplifies to just 0.008 . As assortative mating progresses in successive

347 generations, $R_{t}$ increases, and the mating pool size becomes slightly larger, as follows:

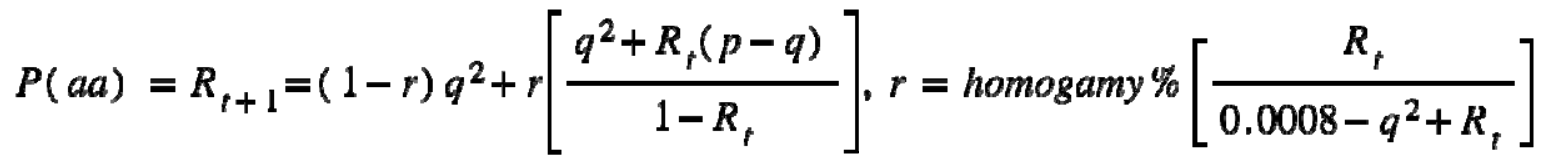

\section{Statistical Testing and Graphing}

350 Statistical comparisons between datasets were performed using SciPy 1.3 .0 . We performed

351 the Shapiro-Wilk test of normality on ending frequencies. Since these ending frequencies were

352 often not normally distributed, and because we additionally wished to test for significant

353 differences in both medians and variances, we used nonparametric tests: the Mann-Whitney $U$

354 test for two independent groups or the Kruskal-Wallis test for $k$ independent groups. Significant

355 Kruskal-Wallis $p$-values were followed by post hoc pairwise Mann-Whitney tests without

356 Bonferroni correction, which is more sensitive than Dunn's test [32]. Figures were generated in

357 Python using matplotlib 3.1.1.

\section{Acknowledgements}

359 We acknowledge Trevor Klemp and Ashley Bergeron for their help on this project during their

360 summer internships. We thank Mohammad Obiedat and Gaurav Arora for critical comments on 361 drafts.

\section{References}

363 1. Bell AG. Memoir: Upon the Formation of a Deaf Variety of the Human Race. Washington 364 (DC): National Academy of Sciences; 1884.

365 2. Greenwald BH. Revisiting Memoir Upon the Formation of a Deaf Variety of the Human 366 Race: Alexander Graham Bell and Deaf Autonomy. In: Greenwald BH, Murray JJ, editors. In Our Own Hands: Essays in Deaf History, 1780-1970. Washington (DC): Gallaudet University Press; 2016. pp. 149-170. 
3. Greenwald BH, Van Cleve JV. 'A Deaf Variety of the Human Race': Historical Memory, Alexander Graham Bell, and Eugenics. The Journal of the Gilded Age and Progressive Era. 2015;14: 28-48.

372 4. Fay EA. Marriages of the Deaf in America. Washington (DC): Volta Bureau; 1898.

373 5. Mendel G. Versuche über Plflanzenhybriden [Experiments in plant hybrizidation].

$374 \quad 1865 ; \mathrm{IV}: 3-47$.

375 6. Ruben RJ. The history of the genetics of hearing impairment. Ann N Y Acad Sci. 2nd ed. Ann N Y Acad Sci; 1991. pp. 6-15. doi:10.1111/j.1749-6632.1991.tb19571.x

7. Yang T, Guo L, Wang L, Yu X. Diagnosis, Intervention, and Prevention of Genetic Hearing Loss. Adv Exp Med Biol. Singapore: Springer Singapore; 2019;1130: 73-92. doi:10.1007/978-981-13-6123-4_5

8. Schimmenti LA, Martinez A, Telatar M, Lai C-H, Shapiro N, Fox M, et al. Infant hearing loss and connexin testing in a diverse population. Genet Med. 2008;10: 517-524. doi:10.1097/GIM.0b013e31817708fa

9. Van Laer L, Coucke P, Mueller RF, Caethoven G, Flothmann K, Prasad SD, et al. A common founder for the 35delG GJB2 gene mutation in connexin 26 hearing impairment. 2001st ed. 2001;38: 515-518. doi:10.1136/jmg.38.8.515

10. Morell RJ, Kim HJ, Hood LJ, Goforth L, Friderici K, Fisher R, et al. Mutations in the connexin 26 gene (GJB2) among Ashkenazi Jews with nonsyndromic recessive deafness. N Engl J Med. 1998;339: 1500-1505.

11. Yan D, Park H-J, Ouyang XM, Pandya A, Doi K, Erdenetungalag R, et al. Evidence of a

13. Wright S. Systems of Mating. II. the Effects of Inbreeding on the Genetic Composition of

14. Crow JF, Felsenstein J. The effects of assortative mating on the genetic composition of a founder effect for the $235 \mathrm{delC}$ mutation of GJB2 (connexin 26) in east Asians. Human Genetics. 2003;114: 44-50. doi:10.1007/s00439-003-1018-1

12. Fisher RA. The correlation between relatives on the supposition of Mendelian inheritance. Trans Roy Soc Edinb. 1918;52: 399-433. population. Eugenics Quarterly. 1968;15: 85-97.

15. Nance WE, Kearsey MJ. Relevance of connexin deafness (DFNB1) to human evolution. Am J Hum Genet. 2004;74: 1081-1087. doi:10.1086/420979

16. Nance WE, Liu XZ, Pandya A. Relation between choice of partner and high frequency of connexin-26 deafness. Lancet. 2000;356: 500-501. doi:10.1016/S0140-6736(00)02565-4 
17. Arnos KS, Welch KO, Tekin M, Norris VW, Blanton SH, Pandya A, et al. A comparative analysis of the genetic epidemiology of deafness in the United States in two sets of pedigrees collected more than a century apart. Am J Hum Genet. 2008;83: 200-207. doi:10.1016/j.ajhg.2008.07.001

18. Schein J, Delk M. The Deaf Population of the United States. Silver Spring (MD): National Association of the Deaf; 1974.

19. Blanton SH, Nance WE, Norris VW, Welch KO, Burt A, Pandya A, et al. Fitness among individuals with early childhood deafness: studies in alumni families from gallaudet university. Annals of Human Genetics. 2010;74: 27-33. doi:10.1111/j.14691809.2009.00553.x

20. Hu DN, Qiu WQ, Wu BT, Fang LZ, Zhou F, Gu YP, et al. Prevalence and genetic aspects of deaf mutism in Shanghai. J Med Genet. 1987;24: 589-592. doi:10.1136/jmg.24.10.589

21. Liu X, Xu L, Zhang S, Xu Y. Epidemiological and genetic studies of congenital profound deafness in the general population of Sichuan, China. Am J Med Genet. 1994;53: 192195. doi:10.1002/ajmg.1320530214

22. Tekin M, Xia XJ, Erdenetungalag R, Cengiz FB, White TW, Radnaabazar J, et al. GJB2 mutations in Mongolia: complex alleles, low frequency, and reduced fitness of the deaf. Annals of Human Genetics. Blackwell Publishing Ltd; 2010;74: 155-164. doi:10.1111/j.1469-1809.2010.00564.x

23. Burke TB, Snoddon K, Wilkinson E. Genetics and Deafness: A View from the Inside. In: Vona B, Haaf T, editors. Genetics of Deafness. S. Karger AG; 2016. pp. 1-8. doi:10.1159/000442334

24. Scully JL, Burke TB. Russia's CRISPR “Deaf Babies”: the Next Genome Editing Frontier? In: Impact Ethics [Internet]. 9 Jul 2019 [cited 29 Jul 2020]. Available: https://impactethics.ca/2019/07/09/russias-crispr-deaf-babies-the-next-genome-editingfrontier/

26. Peng B, Amos CI. Forward-time simulations of non-random mating populations using

25. Peng B, Kimmel M. simuPOP: a forward-time population genetics simulation environment. Bioinformatics. 2005;21: 3686-3687. doi:10.1093/bioinformatics/bti584

435 28. Mahdieh N, Rabbani B. Statistical study of 35delG mutation of GJB2 gene: a meta- 
438 29. Marazita ML, Ploughman LM, Rawlings B, Remington E, Arnos K, Nance W. Genetic epidemiological studies of early-onset deafness in the U.S. school-age population. Am J Med Genet. 1993;46: 486-491.

441 30. Morton CC, Nance WE. Newborn hearing screening--a silent revolution. N Engl J Med. 2006;354: 2151-2164. doi:10.1056/NEJMra050700

443 31. Tran Van Nhieu G, Clair C, Bruzzone R, Mesnil M, Sansonetti P, Combettes L. Connexin-dependent inter-cellular communication increases invasion and dissemination of Shigella in epithelial cells. Nat Cell Biol. 2003;5: 720-726. doi:10.1038/ncb1021

446 32. Conover WJ, Iman RL. On Multiple-Comparisons Procedures. 1979. Report No.: LA7677-MS. 


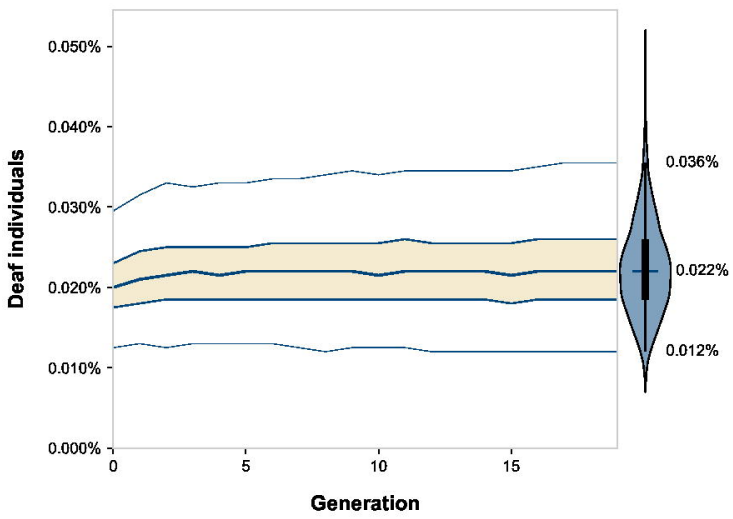




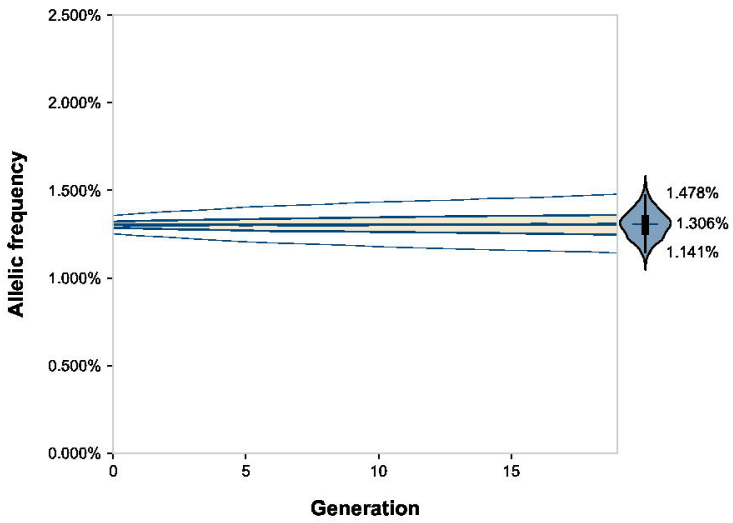




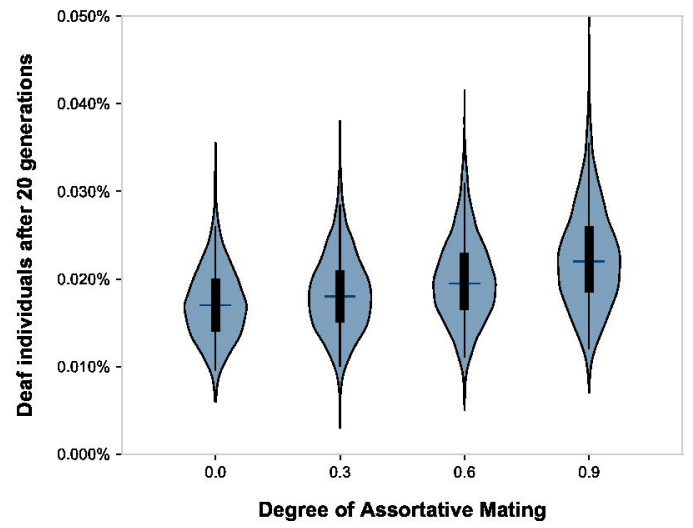




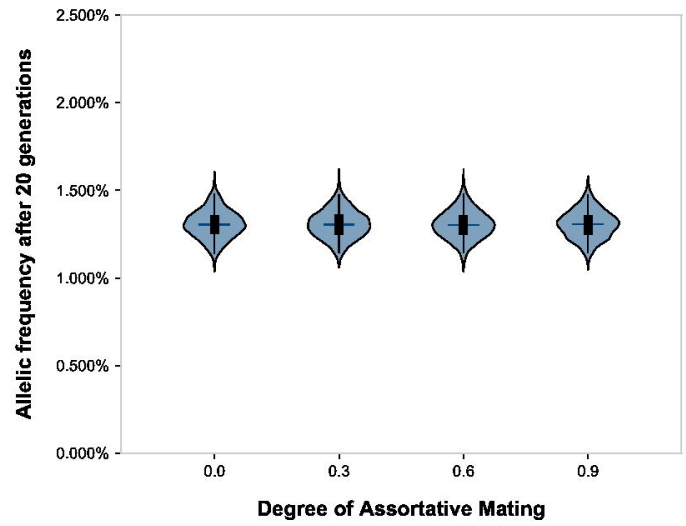




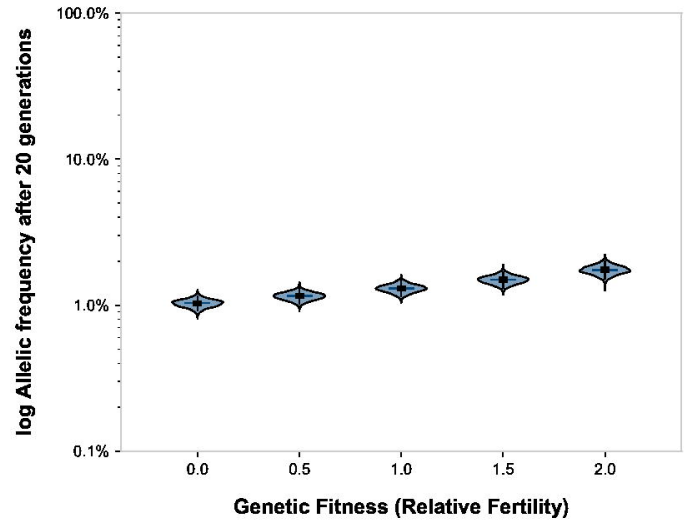




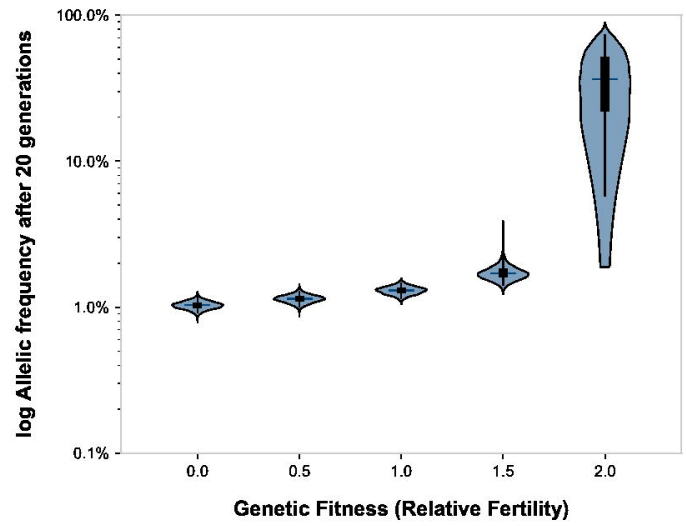

